\title{
GRB 060218 and GRBs associated with supernovae Ib/c
}

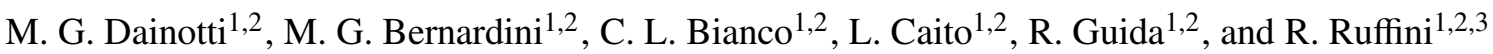 \\ 1 ICRANet and ICRA, Piazzale della Repubblica 10, 65122 Pescara, Italy \\ 2 Dipartimento di Fisica, Università di Roma "La Sapienza", Piazzale Aldo Moro 5, 00185 Roma, Italy \\ e-mail: [dainotti;maria.bernardini; bianco; letizia.caito;roberto.guida;ruffini]@icra.it \\ 3 ICRANet, Université de Nice Sophia Antipolis, Grand Château, BP 2135, 28 avenue de Valrose, 06103 Nice Cedex 2, France
}

Received 12 June 2007 / Accepted 20 June 2007

\section{ABSTRACT}

\begin{abstract}
Context. The Swift satellite has given continuous data in the range $0.3-150 \mathrm{keV}$ from $0 \mathrm{~s}$ to $10^{6} \mathrm{~s}$ for GRB 060218 associated with SN2006aj. This Gamma-Ray Burst (GRB) which has an unusually long duration $\left(T_{90} \sim 2100 \mathrm{~s}\right)$ fulfills the Amati relation. These data offer the opportunity to probe theoretical models for GRBs connected with Supernovae (SNe).

Aims. We plan to fit the complete $\gamma$ - and X-ray light curves of this long duration GRB, including the prompt emission, in order to clarify the nature of the progenitors and the astrophysical scenario of the class of GRBs associated with SNe Ib/c.

Methods. We apply our "fireshell" model based on the formation of a black hole, giving the relevant references. It is characterized by the precise equations of motion and equitemporal surfaces and by the role of thermal emission.

Results. The initial total energy of the electron-positron plasma $E_{\mathrm{e}^{ \pm}}^{\text {tot }}=2.32 \times 10^{50} \mathrm{erg}$ has a particularly low value, similar to the other GRBs associated with SNe. For the first time, we observe a baryon loading $B=10^{-2}$ which coincides with the upper limit for the dynamical stability of the fireshell. The effective CircumBurst Medium (CBM) density shows a radial dependence $n_{\mathrm{cbm}} \propto r^{-\alpha}$ with $1.0 \lesssim \alpha \lesssim 1.7$ and monotonically decreases from 1 to $10^{-6}$ particles $/ \mathrm{cm}^{3}$. This behavior is interpreted as being due to a fragmentation in the fireshell. Analogies with the fragmented density and filling factor characterizing Novae are outlined. The fit presented is particularly significant in view of the complete data set available for GRB 060218 and of the fact that it fulfills the Amati relation. Conclusions. We fit GRB 060218, usually considered as an X-Ray Flash (XRF), as a "canonical GRB" within our theoretical model. The smallest possible black hole, formed by the gravitational collapse of a neutron star in a binary system, is consistent with the especially low energetics of the class of GRBs associated with $\mathrm{SNe} \mathrm{Ib} / \mathrm{c}$. We provide the first evidence for a fragmentation in the fireshell. This fragmentation is crucial in explaining both the unusually large $T_{90}$ and the consequently inferred abnormally low value of the CBM effective density.
\end{abstract}

Key words. gamma rays: bursts - black hole physics - stars: binaries: general - stars: neutron

\section{Introduction}

GRB 060218, discovered by the Swift satellite (Masetti et al. 2006) with cosmological redshift $z=0.033$ (Mirabal et al. 2006; Sollerman et al. 2006), is the best example of a Gamma-Ray Burst (GRB) associated with a Supernova (SN) Ib/c (Campana et al. 2006). Its extremely long duration is unusual, with the longest $T_{90}$ ever observed $\left(T_{90} \sim 2100 \mathrm{~s}\right) . T_{90}$ is defined as the time over which a burst emits from $5 \%$ to $95 \%$ of its total measured energy in the prompt emission. This definition depends however on the instrumental threshold (see Ruffini et al. 2007c, for details). This source is also interesting since it represents a discriminant between existing GRB theories: it has been pointed out by Soderberg et al. (2006b) and Fan et al. (2006) that it is impossible to explain the $\mathrm{X}$ - and radio afterglows within the traditional synchrotron model. They attempted to fit only the late radio data after $\sim 10^{3} \mathrm{~s}$ and they attributed the nature of the prompt emission to an as yet undetermined "inner engine" (see Soderberg et al. 2006b), possibly a magnetar (Mazzali et al. 2006).

Here we present a detailed fit of the entire X-and $\gamma$-ray light curves including the prompt emission: there is no need here for the prolonged activity of an inner engine. Therefore we explain the unusually high values of the observed $T_{90}$ by our "fireshell" model (see Sect. 2 of this paper, Ruffini et al. 2001a,b, 2003, 2005a; Bianco \& Ruffini 2004, 2005a,b, and references therein).
After summarizing our model in Sect. 2, in Sect. 3 we recall GRB 060218's observational data. In Sect. 4 we show the fit of the BAT and XRT light curves (in the $15-150 \mathrm{keV}$ and in the 0.3-10.0 keV energy bands respectively, see Figs. 1, 2). In Fig. 3 and Sect. 5 we discuss the actual and effective CircumBurst Medium (CBM) density. We outline the occurrence of a fragmentation in the fireshell pointing out some analogies with the ejecta of Novae before concluding.

\section{The fireshell model}

We assume that all GRBs, whether "short" or "long", originate from the gravitational collapse of a black hole (Ruffini et al. 2001b). The $\mathrm{e}^{ \pm}$plasma created in the process of the black hole formation expands as a spherically symmetric "fireshell" with a constant width in the laboratory frame, i.e. the frame in which the black hole is at rest. We have only two free parameters characterizing the source, namely the total energy $E_{\mathrm{e}^{ \pm}}^{\text {tot }}$ of the $\mathrm{e}^{ \pm}$ plasma and its baryon loading $B \equiv M_{\mathrm{B}} c^{2} / E_{\mathrm{e}^{ \pm}}^{\text {tot }}$, where $M_{\mathrm{B}}$ is the total baryons' mass (Ruffini et al. 2000). They fully determine the optically thick acceleration phase of the fireshell, which lasts until the transparency condition is reached and the Proper-GRB (P-GRB, Ruffini et al. 2001b) is emitted. The afterglow emission then starts due to the collision between the remaining optically thin fireshell and the CBM, and it clearly depends on the parameters describing the effective CBM distribution (see 
below). The luminosity of such an afterglow emission consists of a rising branch, a peak, and a decaying tail (Ruffini et al. 2001b).

Therefore, unlike treatments in the current literature (e.g. Piran 2004, and references therein), in our model we define a "canonical GRB" light curve with two sharply different components: the P-GRB and the afterglow (Ruffini et al. 2001b, 2007a). The ratio between the total energies of these two components and the temporal separation between their peaks are functions of the $B$ parameter (Ruffini et al. 2001b). The peak of the afterglow contributes to what is usually called the GRB "prompt emission" (e.g. Ruffini et al. 2001b, 2006).

Another crucial assumption is that the afterglow luminosity is due to a thermal emission in the co-moving frame of the fireshell (Ruffini et al. 2004). The $\mathcal{R}$ parameter defines the temperature $T$ of such a thermal emission:

$\mathcal{R} \equiv \frac{A_{\mathrm{eff}}}{A_{\mathrm{vis}}}=\frac{\mathrm{d} E / \mathrm{d} t}{4 \pi r^{2} \sigma T^{4}}$,

where $\mathrm{d} E / \mathrm{d} t$ is the source luminosity, $\sigma$ is the Stefan-Boltzmann constant, $r$ is the radius of the fireshell, $A_{\text {eff }}$ is its effective emitting area and $A_{\text {vis }}$ is its total visible area. $\mathcal{R}$ and the CBM effective density $n_{\mathrm{cbm}}$ are the two parameters which fully describe the effective CBM distribution taking into account its filamentary structure (Ruffini et al. 2005b). Similar considerations in a different context have been recently presented in Pe'er et al. (2007).

The description of the engulfment of the CBM matter by the fireshell is a highly complex and time consuming procedure. In non-relativistic systems such a description can be made at each point. In this ultrarelativistic regime a more global approach is needed. The arrival time of each photon at the detector depends on the entire past history of the fireshell (Ruffini et al. 2001a). All the observables depend on the equitemporal surfaces (EQTSs, Bianco \& Ruffini 2004, 2005a) which produce observable effects in a large part of the future history of the fireshell. The CBM structure due to its feedback on the equation of motion of the fireshell must therefore be inferred self-consistently with this very non-linear evolution of the entire fireshell history. These difficulties are further increased by the necessity to fit the observed light curves in selected energy bands (two in the present case of GRB 060218 and up to five in the case of GRB 050315, Ruffini et al. 2006). The fulfillment of these constraints represents a severe test not only for the validity of the theory but also for the spectral models assumed in the data reduction.

The fireshell model has been successfully applied to GRB 050315 (Ruffini et al. 2006), GRB 031203 (Bernardini et al. 2005), GRB 980425 (Ruffini et al. 2007b), GRB 030329 (Bernardini et al. 2006), GRB 970228 (Bernardini et al., in preparation), GRB 991216 (Ruffini et al. 2003, 2004). Not all these sources fulfill the correlation proposed by Amati et al. (2002) between the isotropic equivalent energy emitted in the prompt emission and the peak energy of the corresponding timeintegrated spectrum (Dainotti et al. in preparation).

\section{GRB 060218 - SN2006aj}

GRB 060218 triggered the BAT instrument of Swift on 18 February 2006 at 03:36:02 UT and has a $T_{90}=(2100 \pm 100) \mathrm{s}$ (Cusumano et al. 2006). The XRT instrument (Kennea et al. 2006; Cusumano et al. 2006) began observations $~ 153$ s after the BAT trigger and continued for $\sim 12.3$ days (Sakamoto et al. 2006). The source is characterized by a flat $\gamma$-ray light curve

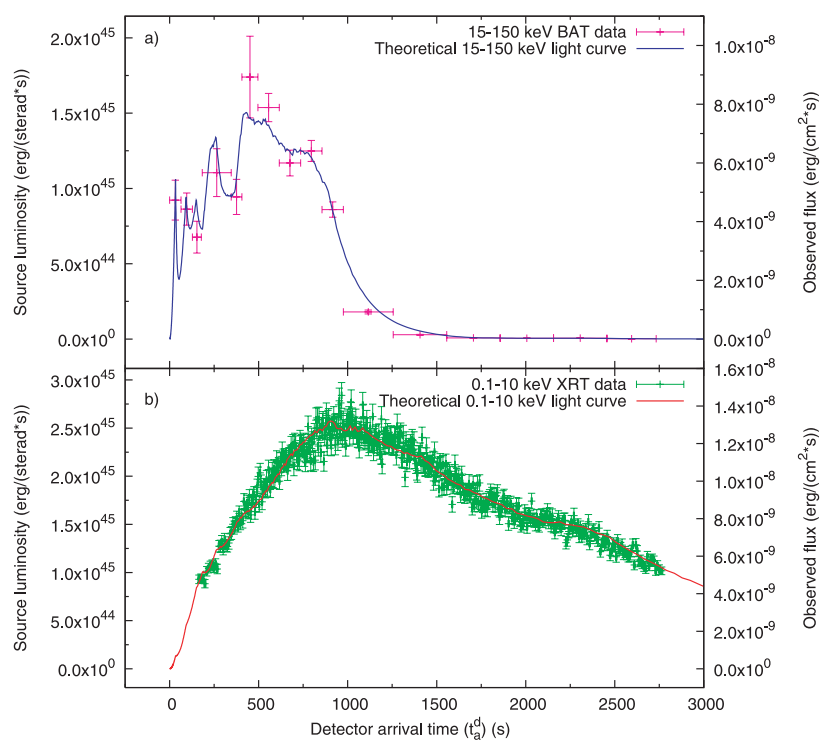

Fig. 1. GRB 060218 prompt emission: a) our theoretical fit (blue line) of the BAT observations in the 15-150 keV energy band (pink points); b) our theoretical fit (red line) of the XRT observations in the $0.3-10 \mathrm{keV}$ energy band (green points) (from Campana et al. 2006).

and a soft spectrum (Barbier et al. 2006). It has an X-ray light curve with a long, slow rise and gradual decline and is considered an X-Ray Flash (XRF) since its peak energy occurs at $E_{\mathrm{p}}=4.9_{-0.3}^{+0.4} \mathrm{keV}$ (Campana et al. 2006). It was observed by the Chandra satellite on February 26.78 and March $7.55 \mathrm{UT}(t \simeq 8.8$ and 17.4 days) for 20 and $30 \mathrm{ks}$ respectively (Soderberg et al. 2006b). The spectroscopic redshift was $z=0.033$ (Sollerman et al. 2006; Mirabal et al. 2006). The corresponding isotropic equivalent energy is $E_{\text {iso }}=(1.9 \pm 0.1) \times 10^{49} \mathrm{erg}$ (Sakamoto et al. 2006) which sets this GRB as having a low luminosity, consistent with most of the GRBs associated with SNe (Liang et al. 2006b; Cobb et al. 2006; Guetta \& Della Valle 2007).

GRB 060218 is associated with SN2006aj whose expansion velocity is $v \sim 0.1 c$ (Pian et al. 2006; Fatkhullin et al. 2006; Soderberg et al. 2006a; Cobb et al. 2006). The host galaxy of SN2006aj is a low luminosity, metal-poor star-forming dwarf galaxy (Ferrero et al. 2006) with an irregular morphology (Wiersema et al. 2007), similar to those of other GRBs associated with SNe (Modjaz et al. 2006; Sollerman et al. 2006).

\section{The fit of the observed data}

In this section we present the fit of our fireshell model to the observed data (see Figs. 1, 2). The fit leads to a total energy of the $\mathrm{e}^{ \pm}$plasma $E_{\mathrm{e}^{ \pm}}^{\text {tot }}=2.32 \times 10^{50} \mathrm{erg}$, with an initial temperature $T=1.86 \mathrm{MeV}$ and a total number of pairs $N_{\mathrm{e}^{ \pm}}=1.79 \times 10^{55}$. The second parameter of the theory, $B=1.0 \times 10^{-2}$, is the highest value ever observed and is close to the limit for the stability of the adiabatic optically thick acceleration phase of the fireshell (for further details see Ruffini et al. 2000). The Lorentz gamma factor, obtained solving the fireshell equations of motion (Bianco $\&$ Ruffini 2005a,b), is $\gamma_{\circ}=99.2$ at the beginning of the afterglow phase at a distance from the progenitor $r_{\circ}=7.82 \times 10^{12} \mathrm{~cm}$. It is much larger than $\gamma \sim 5$ estimated by Kaneko et al. (2006) and Toma et al. (2006).

In Fig. 1 we show the afterglow light curves fitting the prompt emission both in the BAT (15-150 keV) and in the XRT (0.3-10 keV) energy ranges, as expected in our "canonical GRB" scenario (Dainotti et al., in preparation). Initially the 


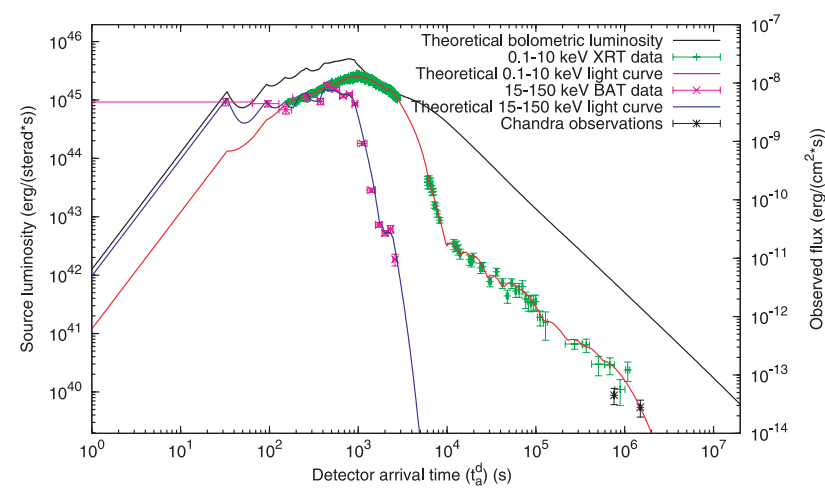

Fig. 2. GRB 060218 complete light curves: our theoretical fit (blue line) of the 15-150 keV BAT observations (pink points), our theoretical fit (red line) of the 0.3-10 keV XRT observations (green points) and the 0.3-10 keV Chandra observations (black points) are represented together with our theoretically computed bolometric luminosity (black line) (from Campana et al. 2006; Soderberg et al. 2006b).

two luminosities are comparable, but for a detector arrival time $t_{\mathrm{a}}^{\mathrm{d}}>1000 \mathrm{~s}$ the XRT curve becomes dominant. The displacement between the peaks of these two light curves leads to a theoretically estimated spectral lag greater than $500 \mathrm{~s}$ in perfect agreement with the observations (see Liang et al. 2006a). We obtain that the bolometric luminosity in this early part coincides with the sum of the BAT and XRT light curves (see Fig. 2) and the luminosity in the other energy ranges is negligible.

We recall that at $t_{\mathrm{a}}^{\mathrm{d}} \sim 10^{4} \mathrm{~s}$ there is a sudden enhancement in the radio luminosity and there is an optical luminosity dominated by the SN2006aj emission (Campana et al. 2006; Soderberg et al. 2006b; Fan et al. 2006). Although our analysis addresses only the BAT and XRT observations, for $r>10^{18} \mathrm{~cm}$ corresponding to $t_{\mathrm{a}}^{\mathrm{d}}>10^{4} \mathrm{~s}$ the fit of the XRT data implies two new features: 1) a sudden increase of the $\mathcal{R}$ factor from $\mathcal{R}=1.0 \times 10^{-11}$ to $\mathcal{R}=1.6 \times 10^{-6}$, corresponding to a significantly more homogeneous effective CBM distribution (see Fig. 3b); 2) an XRT luminosity much smaller than the bolometric one (see Fig. 2). These theoretical predictions may account for the energetics of the enhancement of the radio and possibly optical and UV luminosities. Therefore, we identify two different regimes in the afterglow, one for $t_{\mathrm{a}}^{\mathrm{d}}<10^{4} \mathrm{~s}$ and the other for $t_{\mathrm{a}}^{\mathrm{d}}>10^{4} \mathrm{~s}$. Nevertheless, there is a unifying feature: the determined effective CBM density decreases with the distance $r$ monotonically and continuously through both these two regimes from $n_{\mathrm{cbm}}=1$ particle $/ \mathrm{cm}^{3}$ at $r=r_{\text {。 }}$ to $n_{\mathrm{cbm}}=10^{-6}$ particle $/ \mathrm{cm}^{3}$ at $r=6.0 \times 10^{18} \mathrm{~cm}: n_{\mathrm{cbm}} \propto r^{-\alpha}$, with $1.0 \lesssim \alpha \lesssim 1.7$ (see Fig. 3a).

Our assumption of spherical symmetry is supported by the observations which set for GRB 060218 an opening beaming angle larger than $\sim 37^{\circ}$ (Liang et al. 2006b; Campana et al. 2006; Soderberg et al. 2006b; Guetta \& Della Valle 2007).

\section{The fireshell fragmentation}

GRB 060218 presents different peculiarities: the extremely long $T_{90}$, the very low effective CBM density decreasing with distance and the largest possible value of $B=10^{-2}$. These peculiarities appear to be correlated. Following Ruffini et al. (2007b), we propose that in the present case the fireshell is fragmented. This implies that the surface of the fireshell does not increase

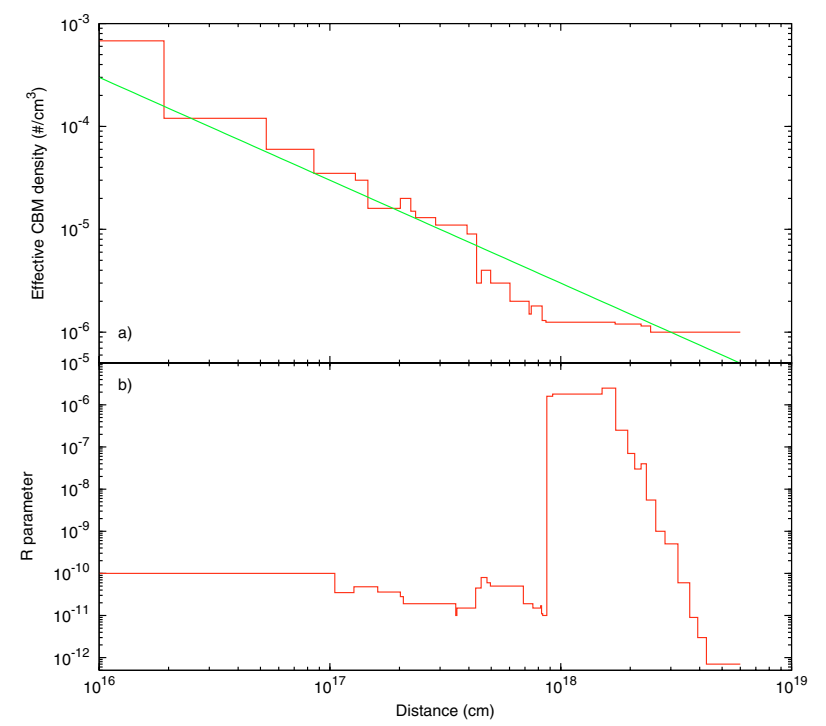

Fig. 3. The CBM distribution parameters: a) the effective CBM number density (red line) monotonically decreases with the distance $r$ following Eq. (2) (green line); b) the $\mathcal{R}$ parameter vs. distance.

further as $r^{2}$ but as $r^{\beta}$ with $\beta<2$. Consequently, the effective $\mathrm{CBM}$ density $n_{\mathrm{cbm}}$ is linked to the actual $n_{\mathrm{cbm}}^{\text {act }}$ by:

$n_{\mathrm{cbm}}=\mathcal{R}_{\mathrm{shell}} n_{\mathrm{cbm}}^{\mathrm{act}}, \quad$ with $\quad \mathcal{R}_{\text {shell }} \equiv\left(r^{\star} / r\right)^{\alpha}$,

where $r^{\star}$ is the starting radius at which fragmentation occurs and $\alpha=2-\beta$ (Fig. 3a). For $r^{\star}=r_{\circ}$ we have $n_{\text {cbt }}^{\text {act }}=1$ particles $/ \mathrm{cm}^{3}$, as expected for a "canonical GRB" (Ruffini et al. 2007a) and in agreement with the apparent absence of a massive stellar wind in the CBM (Soderberg et al. 2006b; Fan et al. 2006; Li 2007).

The $\mathcal{R}$ parameter defined in Eq. (1) has to take into account both the effect of the fireshell fragmentation $\left(\mathcal{R}_{\text {shell }}\right)$ and of the effective CBM porosity $\left(\mathcal{R}_{\mathrm{cbm}}\right)$ :

$\mathcal{R} \equiv \mathcal{R}_{\text {shell }} \times \mathcal{R}_{\text {cbm }}$.

The phenomenon of the clumpiness of the ejecta, whose measure is the filling factor, is a well known aspect in astrophysics. For example, in the case of Novae the filling factor has been measured to be in the range $10^{-2}-10^{-5}$ (Ederoclite et al. 2006). Such a filling factor coincides, in our case, with $\mathcal{R}_{\text {shell }}$.

\section{Binaries as progenitors of GRB-SN systems}

The majority of the existing models in the literature appeal to a single astrophysical phenomenon to explain both the GRB and the SN ("collapsar", e.g. Woosley \& Bloom 2006). On the contrary, a distinguishing feature of our theoretical approach is to differentiate between the SN and the GRB process. The GRB is assumed to occur during the formation process of a black hole. The $\mathrm{SN}$ is assumed to lead to the formation of a neutron star (NS) or to a complete disruptive explosion without remnants and, in no way, to the formation of a black hole. In the case of SN2006aj the formation of such a NS has been inferred by Maeda et al. (2007) because of the large amount of ${ }^{58} \mathrm{Ni}$ $\left(0.05 M_{\odot}\right)$. Moreover the significantly small initial mass of the SN progenitor star $M \approx 20 M_{\odot}$ is expected to form a NS rather than a black hole when its core collapses (Maeda et al. 2007; Ferrero et al. 2006; Mazzali et al. 2006; Nomoto et al. 2007). In order to fulfill both the above requirement, we assume that the progenitor of the GRB and the SN consists of a binary system formed by a NS close to its critical mass collapsing to a 
black hole, and a companion star evolved out of the main sequence originating the SN. The temporal coincidence between the GRB and the SN phenomenon is explained in terms of the concept of "induced" gravitational collapse (Ruffini et al. 2001c, 2007b). There is also the distinct possibility of observing the young born NS out of the SN (e.g. Ruffini et al. 2007b, and references therein).

It has been often proposed that GRBs associated with $\mathrm{SNe}$ $\mathrm{Ib} / \mathrm{c}$, at smaller redshift $0.0085<z<0.168$ (e.g. Della Valle 2006, and references therein), form a different class, less luminous and possibly much more numerous than the high luminosity GRBs at higher redshift (Pian et al. 2006; Soderberg et al. 2004; Maeda et al. 2007; Della Valle 2006). Therefore they have been proposed to originate from a separate class of progenitors (Liang et al. 2006b; Cobb et al. 2006). In our model this is explained by the nature of the progenitor system leading to the formation of the black hole with the smallest possible mass: that formed by the collapse of a just overcritical NS (Ruffini 2006; Ruffini et al. 2007b).

The recent observation of GRB 060614 at $z=0.125$ without an associated SN (Della Valle et al. 2006; Mangano et al. 2007) gives strong support to our scenario, alternative to the collapsar model. Also in this case the progenitor of the GRB appears to be a binary system composed of two NSs or a NS and a white dwarf (Caito et al., in preparation).

\section{Conclusions}

GRB 060218 presents a variety of peculiarities, including its extremely large $T_{90}$ and its classification as an XRF. Nevertheless, a crucial point of our analysis is that we have successfully applied to this source our "canonical GRB" scenario.

Within our model there is no need for inserting GRB 060218 in a new class of GRBs, such as the XRFs, alternative to the "canonical" ones. This same point recently received strong observational support in the case of GRB 060218 (Liang et al. 2006a) and a consensus by other models in the literature (Kaneko et al. 2006).

The anomalously long $T_{90}$ led us to infer a monotonic decrease in the CBM effective density providing the first clear evidence for fragmentation in the fireshell. This phenomenon appears to be essential in understanding the features of other GRBs (e.g. GRB 050315 in Ruffini et al. 2007b, and GRB 970228 in Bernardini et al. in preparation).

Our "canonical GRB" scenario originates from the gravitational collapse to a black hole and is now confirmed over a $10^{6}$ range in energy (e.g. Ruffini et al. 2007a, and references therein). It is clear that, although the process of gravitational collapse is unique, there is a large variety of progenitors which may lead to the formation of black holes, each one with precise signatures in the energetics. The low energetics of the class of GRBs associated with $\mathrm{SNe}$, and the necessity of the occurrence of the $\mathrm{SN}$, naturally leads in our model to the identification of their progenitors with the formation of the smallest possible black hole originating from a NS overcoming its critical mass in a binary system. For GRB 060218 there is no need within our model for a new or unidentified source such as a magnetar or a collapsar.

GRB 060218 is the first GRB associated with SN with complete coverage of data from the onset up to $\sim 10^{6} \mathrm{~s}$. This fact offers an unprecedented opportunity to verify theoretical models on such a GRB class. For example, GRB 060218 fulfills the Amati et al. (2002) relation unlike other sources in its same class. This is particularly significant, since GRB 060218 is the only source in such a class to have an excellent data coverage without gaps. We are currently examining if the missing data in the other sources of such a class may have a prominent role in their nonfulfillment of the Amati et al. (2002) relation (Dainotti et al., in preparation; Ghisellini et al. 2006).

Acknowledgements. We thank the Italian Swift Team (supported by ASI Grant I/R/039/04 and partly by the MIUR grant 2005025417) for the reduced Swift data, and Michael Kramer and Nino Panagia for the wording of the manuscript.

\section{References}

Amati, L., Della Valle, M., Frontera, F., et al. 2002, A\&A, 390, 81 Barbier, L., Barthelmy, S., Cummings, J., et al. 2006, GCN Circ., 4780 Bernardini, M. G., Bianco, C. L., Chardonnet, P., et al. 2005, ApJ, 634, L29 Bernardini, M. G., Bianco, C. L., Chardonnet, P., et al. 2006, in Proceedings of the Xth Marcel Grossmann Meeting (World Scientific), 2459

Bianco, C. L., \& Ruffini, R. 2004, ApJ, 605, L1

Bianco, C. L., \& Ruffini, R. 2005a, ApJ, 620, L23

Bianco, C. L., \& Ruffini, R. 2005b, ApJ, 633, L13

Campana, S., Mangano, V., Blustin, A. J., et al. 2006, Nature, 442, 1008

Cobb, B. E., Bailyn, C. D., van Dokkum, P. G., \& Natarajan, P. 2006, ApJ, 645, L113

Cusumano, G., Barthelmy, S., Gehrels, N., et al. 2006, GCN Circ., 4775 Della Valle, M. 2006, AIP Con. Proc. 836, 367

Della Valle, M., Chincarini, G., Panagia, N., et al. 2006, Nature, 444, 1050 Ederoclite, A., Mason, E., Della Valle, M., et al. 2006, A\&A, 459, 875 Fatkhullin, T. A., Vlasyuk, V. V., Sokolov, V. V., et al. 2006, GCN Circ., 4809 Fan, Y., Piran, T., \& Xu, D. 2006, JCAP, 0609, 013

Ferrero, P., Palazzi, E., Pian, E., \& Savaglio, S., in Proceedings of The Multicoloured Landscape of Compact Objects and their Explosive Progenitors: Theory vs Observations [arXiv:astro-ph/0610417] Ghisellini, G., Ghirlanda, G., Mereghetti, S., et al. 2006, MNRAS, 372, 1699 Guetta, D., \& Della Valle, M. 2007, ApJ, 657, L73

Kaneko, Y., Ramirez-Ruiz, E., Granot, J., et al. 2006, ApJ, 654, 385

Kennea, J., Burrows, D., Cusumano, G., \& Tagliaferri, G. 2006, GCN Circ., 4776 Liang, E. W., Zhang, B.-B., Stamatikos, M., et al. 2006a, ApJ, 653, L81

Liang, E., Zhang, B., Virgili, F., \& Dai, Z. G. 2006b

[arXiv: astro-ph/0605200]

Li, L.-X. 2007, MNRAS, 375, 240

Mangano, V., Holland, S. T., Malesani, D., et al. 2007 [arXiv: 0704 .2235]

Masetti, N., Palazzi, E., Pian, E., \& Patat, F. 2006, GCN Circ., 4803

Maeda, K., Kawabata, K., Tanaka, M., et al. 2007, ApJ, 658, L5

Mazzali, P., Deng, J., Nomoto, K., et al. 2006, Nature, 442, 1018

Mirabal, N., Halpern, J., Thorstensen, J., \& Terndrup, D. 2006, ApJ, 643, L99

Modjaz, M., Stanek, K. Z., Garnavich, P. M., et al. 2006, ApJ, 645, L21

Nomoto, K., Tominaga, N., Tanaka, M., et al. 2007

[arXiv: astro-ph/0702472]

Pe'er, A., Ryde, F., Wijers, R. A. M. J., et al. 2007 [arXiv: astro-ph/0703734]

Pian, E., Mazzali, P., Masetti, N., et al. 2006, Nature, 442, 1011

Piran, T. 2004, Rev. Mod. Phys., 76, 1143

Ruffini, R. 2006, in the Proceedings of the XIth Marcel Grossmann meeting (World Scientific), in press

Ruffini, R., Salmonson, J. D., Wilson, J. R., \& Xue, S. S. 2000, A\&A, 359, 855 Ruffini, R., Bianco, C. L., Chardonnet, P., et al. 2001a, ApJ, 555, L107 Ruffini, R., Bianco, C. L., Chardonnet, P., et al. 2001b, ApJ, 555, L113 Ruffini, R., Bianco, C. L., Chardonnet, P., et al. 2001c, ApJ, 555, L117 Ruffini, R., Bianco, C. L., Chardonnet, P., et al. 2003, AIP Con. Proc., 668, 16 Ruffini, R., Bianco, C. L., Chardonnet, P., et al. 2004, Int. J. Mod. Phys. D, 13, 843

Ruffini, R., Bernardini, M. G., Bianco, C. L., et al. 2005a, AIP Con. Proc., 782, 42

Ruffini, R., Bianco, C. L., Chardonnet, P., et al. 2005b, Int. J. Mod. Phys. D, 14, 97

Ruffini, R., Bernardini, M. G., Bianco, C. L., et al. 2006, ApJ, 645, L109 Ruffini, R., Bernardini, M. G., Bianco, C. L., et al. 2007a, AIP Con. Proc., 910, 55

Ruffini, R., Bernardini, M. G., Bianco, C. L., et al. 2007b [arXiv:0705.2456] Ruffini, R., Bernardini, M. G., Bianco, C. L., et al. 2007c [arXiv: 0705 . 2453] Sakamoto, T., Barbier, L., Barthelmy, S., et al. 2006, GCN Circ., 4822 Soderberg, A. M., Kulkarni, S. R., Berger, E., et al. 2004, Nature, 430, 648 Soderberg, A. M., Berger, E., Schmidt, B. P., et al. 2006a, GCN Circ., 4804 Soderberg, A. M., Kulkarni, S. R., Nakar, E., et al. 2006b, Nature, 442, 1014 Sollerman, J., Jaunsen, A. O., \& Fynbo, J. P. U., et al. 2006, A\&A, 454, 503 Toma, K., Ioka, K., Sakamoto, T., \& Nakamura, T. 2007, ApJ, 659, 1420 Wiersema, K., Savaglio, S., Vreeswijk, P. M., et al. 2007, A\&A, 464, 529 Woosley, S. E., \& Bloom, J. S. 2006, ARA\&A, 44, 507 